This is an electronic version of an article published as: Penner, A.R. (2012). Induced energy polarization of the vacuum as the source of the baryonic Tully-Fisher relationship and the Pioneer anomaly.

Canadian Journal of Physics, 90(4), 315-321. DOI: 10.1139/P2012-029

Canadian Journal of Physics is available online at: http://www.nrcresearchpress.com/journal/cjp and this article is available at: http://dx.doi.org/10.1139/P2012-029 


\title{
Induced energy polarization of the vacuum as the source of the baryonic Tully-Fisher relationship and the Pioneer anomaly
}

\author{
Author: $\quad$ A Raymond Penner \\ Address: Department of Physics, \\ Vancouver Island University, \\ 900 Fifth Street, \\ Nanaimo, BC, Canada, \\ V9R 5S5 \\ Email: $\quad$ raymond.penner@ viu.ca \\ Tel: $\quad 250753-3245$ ext: 2336 \\ Fax: $\quad 250740-6482$
}

PACS number: 90.95.30.-k 


\begin{abstract}
A theory where the gravitational field of a mass induces a gravitational field contribution from the cosmos is presented. In this theory, entities of both net positive and net negative energy come into existence throughout the cosmos with a maximum lifetime in accordance with Heisenberg's uncertainty principle. A model of the resulting contribution that these entities make to the gravitational field is provided and is shown to lead to both the baryonic Tully-Fisher relationship and the Pioneer anomaly.
\end{abstract}

\title{
I Introduction
}

Newton's gravitational theory has been used for centuries to predict the motion of objects within our solar system. In Newton's theory, the gravitational field, $\mathbf{g}_{\mathbf{M}}$, at a distance $r$ from a given mass $\mathrm{M}$ points towards the mass and has a magnitude given by

$$
\mathrm{g}_{\mathrm{M}}=\frac{\mathrm{GM}}{\mathrm{r}^{2}}
$$

where G is Newton's gravitational constant. Newton's theory has been extremely successful, but over the years anomalies have been observed. For example, observational data indicated that the orbit of Uranus deviated from what was predicted by Newton's theory. One possibility, considered at the time, was that Newton's theory broke down at great distances from the Sun. However, the subsequent discovery of Neptune accounted for this discrepancy and Newton's gravitational theory was put on even firmer footing. Another historical example was the anomalous precession of the perihelion of Mercury. Again, observational data indicated a deviation from what was predicted by Newton's gravitational theory. However, in this case no additional planet was found and Newton's gravitational theory did indeed break down. A new gravitational theory was required, Einstein's general theory of relativity, to explain the anomaly.

Currently, there are new anomalies that require explanation. One of these, found within our own solar system, is referred to as the Pioneer anomaly as it stems from the analysis of tracking data from the Pioneer 10 and 11 spacecrafts [1-3]. This data has indicated the onset of an anomalous acceleration of the spacecraft directed towards the Sun at a distance between 10 to $15 \mathrm{AU}\left(1 \mathrm{AU}=1.49597870 \times 10^{11} \mathrm{~m}\right)$. From a distance of 15 AU to the limits of the data, approximately $50 \mathrm{AU}$ from the Sun, this anomalous acceleration appears to be constant with a value of

$$
\mathrm{a}_{\text {PIONEER }}=(8.74 \pm 1.33) \times 10^{-10} \mathrm{~m} \mathrm{~s}^{-2} \text {. }
$$

Over this distance the gravitational field of the Sun varies from $2.6 \times 10^{-5} \mathrm{~m} \mathrm{~s}^{-2}$ to $2.4 \times 10^{-6} \mathrm{~m} \mathrm{~s}^{-2}$ so the effect is relatively small. For distances within $15 \mathrm{AU}$ of the Sun, the uncertainties in the data are large but the results do seem to indicate that the anomalous acceleration is not present 
within the inner solar system (i.e., < 10 - 15 AU from the Sun). This agrees with planetary ephemerides and the results from the Viking mission [2] and the Cassini mission [4] which indicate that at least within the inner solar system no anomalous acceleration seems to exist.

The Pioneer anomaly is a much more contentious anomaly than those that were associated with the orbits of Uranus or Mercury. Although attempts to explain the anomalous acceleration as a result of forces generated by the spacecrafts themselves [3] have not yielded values of large enough magnitude, much of the scientific community still does not accept the Pioneer anomaly as pointing the way to new discoveries.

A second observation that disagrees with accepted gravitational theory is that the orbital velocities of galaxies are found to be much greater than what is predicted. There are two general characteristics of this anomaly. First, it is found that the galactic orbital velocities do not fall off with increasing distance as current gravitational theory predicts. But, remarkably, as one moves further from the galactic center the rotational velocity curves flatten out and the rotational velocity remains relatively constant. Secondly, there is a relationship between this constant orbital velocity found at large $r$ and the total luminosity of the galaxy. This is called the TullyFisher [5] relationship. By taking into account the gas content of galaxies it has been shown [6] that this relationship is fundamentally a relationship between $\mathrm{v}$, the rotational velocity of the galaxy, and M, the total baryonic mass of the galaxy. This baryonic Tully-Fisher relationship is given by

$$
\mathrm{M}=35 \mathrm{M}_{\mathrm{S}}(\mathrm{v}\{\mathrm{km} / \mathrm{s}\})^{4}
$$

where $\mathrm{M}_{\mathrm{S}}$ is the mass of the Sun.

As an explanation for the larger than predicted galactic rotational velocities, the assumption that the mass of galaxies resides primarily in the observable luminous matter, namely the stars, was brought into question [7]. A model of dark matter has thereby arisen wherein it is currently believed that the major contribution to the mass of a galaxy, and thereby its gravitational field, are undetected nonbaryonic particles. Other explanations for the observed orbital velocities of galaxies have been proposed. These include Modified Newtonian Dynamics (MOND), as proposed by Milgrom [8-12], which postulates that the inertia of an object varies with acceleration and modified gravitation (MOG), as proposed by Moffat [13-17].

One alternative to the dark matter theory, as proposed by Blanchet [18-20], that is pertinent to the present paper is the existence of a cosmic medium consisting of gravitational dipoles that become polarized in a gravitational field. This theory differs from both MOND and theories such as MOG in that no modification of current gravitational theory or Newtonian dynamics is required. The polarized dipole medium provides an additional contribution to the gravitational field surrounding a given mass. This model is in some sense similar to the dark matter model in that nonbaryonic particles, in this case gravitational dipoles, contribute to the gravitational field of a given mass. However, a major difference between this polarized cosmic 
medium model and the dark matter theory is that the field contribution of the gravitational dipoles, unlike dark matter particles, is induced by the gravitational field of a given mass.

The gravitational dipoles in Blanchet's theory have been modeled [18] as consisting of a pair of particles, one of positive gravitational mass and the other of negative gravitational mass, with both particles having positive inertial mass. The behavior of these gravitational dipoles in a gravitational field is treated as being analogous to electric dipoles in an electric field in that their dipole moments align with the gravitational field. An unspecified internal force between the pair of particles that constitute the dipole is required to bind the dipole together. This force is also required to balance the local gravitational field so that the dipole distribution about a given mass is stable. The internal force, and therefore the dipole moment, is modeled as either being dependent on the gravitational field [18,19] or on the polarization field [20]. The dependence is such that the model leads to the baryonic Tully-Fisher relationship.

Penner [21] considered the general case in which the gravitational field of a mass induces a gravitational contribution, $\mathrm{g}_{\mathrm{A}}$, from the surrounding vacuum. It was shown that, irrespective of the details of the model, the relationship between the induced gravitational field and the total gravitational field, $\mathrm{g}=\mathrm{g}_{\mathrm{A}}+\mathrm{g}_{\mathrm{M}}$, can be expressed as

$$
\frac{\mathrm{g}_{\mathrm{A}}}{\mathrm{g}_{0}}=\frac{\mathrm{g}}{\mathrm{g}_{0}}-\mathrm{k}\left(\frac{\mathrm{g}}{\mathrm{g}_{0}}\right)^{2}+\mathcal{O}\left[\left(\frac{\mathrm{g}}{\mathrm{g}_{0}}\right)^{3}\right]
$$

where $\mathrm{k}$ and $\mathrm{g}_{0}$ are constants. From equation (4), in the weak gravitational field limit, Penner [21] then derived the following equation,

$$
\mathrm{M}=\frac{\mathrm{k}}{\mathrm{Gg}_{0}} \mathrm{v}^{4},
$$

which is in agreement with the baryonic Tully-Fisher relationship. In addition, it was shown that in the strong field limit a value for $\mathrm{g}_{\mathrm{A}}$ would be obtained that is in reasonable agreement with the observed Pioneer anomaly. Examples of possible functions for $\mathrm{g}_{\mathrm{A}}(\mathrm{g})$ were presented, but Penner [21] did not derive any specific relationship between $g_{A}$ and $g$.

Penner [21] also offered an alternative to Blanchet's theory on the manner in which the gravitational field of a mass may induce a gravitational field from the vacuum. In Penner's model, entities of both net positive and net negative energy continually come into existence in the vacuum with a maximum lifetime dictated by Heisenberg's uncertainty principle. The positive energy entities accelerate towards the given mass during their lifetime, while the negative energy entities accelerate away. In this model each displaced entity is treated as equivalent to an energy dipole. Unlike Blanchet's model, these positive and negative entities are not bound to each other and no force is required to keep their distribution stable. The finite lifetime of the entities in Penner's model ensures a time-independent distribution. 
This present paper is a continuation of Penner [21]. A model of the behaviour of the positive energy and negative energy entities in a gravitational field will be presented and the specific relationship between the resulting induced gravitational field $g_{A}$ and the total gravitational field $g$ will be derived. The resulting relationship will be found to lead to both the baryonic Tully-Fisher relationship and the Pioneer anomaly.

\section{Model}

In Penner [21] it is hypothesised that throughout the cosmos entities of both net positive energy and net negative energy continuously come into and out of existence. The maximum lifetime, $\tau$, of these entities is given by the Heisenberg uncertainty principle;

$$
\tau \cong \frac{\hbar}{2|\mathrm{E}|}
$$

where $\mathrm{E}$ is the net energy of a given entity.

Given the above hypothesis, consider the effect that a gravitational field will have on these entities. An entity of net positive energy will during its lifetime be attracted towards the source of the gravitational field. Similarly, an entity of net negative energy will be repelled away from the source. The behaviour of the vacuum in the vicinity of a mass is therefore analogous to some degree to the behaviour of a dielectric in the vicinity of a charge. In the case of a dielectric in the presence of a positive charge, negative charge within the dielectric will shift towards the positive charge and concurrently positive charge will shift away. Overall, the net charge of the dielectric remains zero. The dielectric therefore becomes charge polarized when placed in the vicinity of a charge. The charge polarized dielectric creates an electric field that opposes the electric field due to the charge and as a result the net electric field surrounding a charge placed in a dielectric is reduced. In the case of the vacuum surrounding a mass, positive energy entities within the vacuum will shift towards the mass and concurrently negative energy entities will shift away while the net energy of the vacuum remains zero. The vacuum thereby becomes energy polarized in the vicinity of a mass. The energy polarized vacuum will contribute a gravitational field that adds to the gravitational field of the mass causing the net gravitational field surrounding a mass to be increased.

The above behaviour proposed for the net negative energy entities does require a modification to Newton's $2^{\text {nd }}$ law. Specifically that $m_{I}$, the inertia mass of an entity, needs to be given by

$$
\mathrm{m}_{\mathrm{I}}=\frac{|\mathrm{E}|}{\mathrm{c}^{2}}
$$

where $|E|$ is the magnitude of the total energy of the given entity. The repulsive gravitational force exerted by the source of the gravitational field on a net negative energy entity will thereby result in the entity accelerating away from the source. 
In Penner [21], it is shown that the resulting induced or anomalous gravitational field (anomalous referring to the gravitational field that is in addition to what current gravitational theory predicts) due to the energy polarization of the vacuum is given by

$$
\mathbf{g}_{\mathrm{A}}=\frac{4 \pi \mathrm{G}}{\mathrm{c}^{2}} \mathbf{P}_{\mathrm{E}}
$$

where $\mathrm{P}_{\mathrm{E}}$ is the equivalent energy dipole moment density. The equivalent energy dipole moment density will be given by

$$
\mathbf{P}_{\mathrm{E}}=\mathrm{N} \overline{\mathbf{p}_{\mathrm{E}} \mathrm{t}_{\mathrm{E}}}
$$

where $\mathrm{N}$ is the rate per unit volume at which entities (both positive energy and negative energy) come into existence, $\mathbf{p}_{\mathrm{E}}$ is the equivalent energy dipole moment of a given entity, and $t_{\mathrm{E}}$ is the lifetime of a given entity. The lifetime of a given entity, $t_{\mathrm{E}}$, will in general be less than $\tau$, its maximum possible lifetime, as a result of interactions. The bar over $\mathbf{p}_{\mathbf{E}} \mathbf{t}_{\mathrm{E}}$ and over subsequent quantities represents an averaging over the entities. It is hypothesised that these entities come into existence with zero velocity with respect to the gravitational field and therefore with zero velocity with respect to the source of the gravitational field. It is further hypothesized that these entities come into existence as a pair with one of the entities having a net energy of $+\mathrm{E}_{1}$ while the other has a net energy of $-\mathrm{E}_{1}$. These two entities are taken to be unbound and therefore as a result of the gravitational field the $+\mathrm{E}_{1}$ entities will accelerate towards the source of the gravitational field while the $-\mathrm{E}_{1}$ entities will accelerate away. The equivalent energy dipole moment for any given entity will thereby be given by

$$
\mathbf{p}_{\mathbf{E}}=\mathrm{E}\langle\mathbf{x}\rangle_{\mathrm{t}}
$$

where $\mathrm{E}$ is the net energy of the entity, either $\pm \mathrm{E}_{1}$, and $\langle\mathrm{x}\rangle_{\mathrm{t}}$ is the time-averaged displacement of the entity due to the gravitational field. The equivalent energy dipole moment for a given entity will point towards the source of the gravitational field for entities of both positive energy and negative energy (in this case $\mathrm{E}$ is negative and $\langle\mathbf{x}\rangle_{\mathrm{t}}$ points away from the mass). The gravitational field due to the equivalent energy dipoles, as given by equation (8), will therefore add to the gravitational field of the mass and the magnitude of the total gravitational field $g$ surrounding a mass will be given by

$$
\mathrm{g}=\mathrm{g}_{\mathrm{M}}+\mathrm{g}_{\mathrm{A}}
$$

The magnitude of the time-averaged displacement of an entity over its lifetime will be given by

$$
\langle\mathrm{x}\rangle_{\mathrm{t}}=\frac{1}{\mathrm{t}_{\mathrm{E}}} \int_{0}^{\mathrm{t}_{\mathrm{E}}}\left(\frac{1}{2} \mathrm{gt}^{2}\right) \mathrm{dt}
$$


The value of the gravitational field that is used in equation (12) should properly be the local gravitational field at the location of the entity. The local gravitational field is equal to the total gravitational field minus the minus the contribution that the entity itself contributes to the calculated value for $g$. An analogous correction is required when calculating the electric field that acts on an electric dipole within a dielectric [22]. However, in order to simplify the derivation the local gravitational field will be taken to be equal to the total gravitational field $g$ at that location. Integrating equation (12) then results in

$$
\begin{aligned}
\langle\mathrm{x}\rangle_{\mathrm{t}} & =\frac{1}{3}\left(\frac{1}{2} \mathrm{~g} \mathrm{t}_{\mathrm{E}}^{2}\right) \\
& =\frac{1}{3} \mathrm{x}_{\mathrm{A}}
\end{aligned}
$$

where $\mathrm{x}_{\mathrm{A}}$, the magnitude of the net displacement of an entity, is given by

$$
\mathrm{x}_{\mathrm{A}}=\frac{1}{2} \mathrm{~g} \mathrm{t}_{\mathrm{E}}^{2}
$$

By equations (8), (9), (10), and (13b), the anomalous gravitational field due to the entities can be expressed as

$$
g_{A}=\frac{4 \pi G}{3 c^{2}} N E_{1} \overline{x_{A} t_{E}}
$$

Substituting for $t_{\mathrm{E}}$ from equation (14) then leads to

$$
g_{A}=\frac{4 \pi G}{3 c^{2}} N E_{1} \sqrt{\frac{2}{g}} \overline{x_{A}^{3 / 2}}
$$

The value of $\overline{\mathrm{x}_{\mathrm{A}}{ }^{3 / 2}}$ can be determined from the probability function, $\mathrm{P}(\mathrm{x})$, for the distance travelled by an entity before interacting with another entity. Irrespective of the details of the interaction this probability function $\mathrm{P}(\mathrm{x})$ will be given by the Beer-Lambert law;

$$
P(x)=\mu e^{-\mu x}
$$

where $\mu$, the attenuation coefficient, is given by

$$
\mu=\sigma n_{i}
$$

with $\sigma$ being the cross section for the interaction and $n_{i}$ is the number density of the entities, i.e. the number of entities per unit volume, that can take part in an interaction. The attenuation coefficient can also be expressed by

$$
\mu=\frac{1}{\mathrm{~L}}
$$


where $\mathrm{L}$ is the mean free path length for the entities. To determine $\mathrm{n}_{\mathrm{i}}$ it will be taken that $\mathrm{N}$, the rate per unit volume at which the entities come into existence, is constant and that the only interaction that is of concern is when an entity of energy $+\mathrm{E}_{1}$ interacts with an entity of $-\mathrm{E}_{1}$ resulting in the disappearance of both entities. As such, the number density of the entities that can take part in an interaction with an entity of opposite energy will be given by

$$
\mathrm{n}_{\mathrm{i}}=\frac{1}{2} \mathrm{~N} \overline{\mathrm{t}_{\mathrm{E}}}
$$

and the attenuation coefficient, by equation (18), will thereby be

$$
\mu=\frac{1}{2} \mathrm{~N} \sigma \overline{\mathrm{t}} \overline{\mathrm{E}} \text {. }
$$

Using the Beer-Lambert law the average displacement of an entity can be determined. If the distance travelled by a given entity is solely dictated by how far it travels before an interaction, the average displacement of an entity before it would interact with an entity of opposite energy would be given by

$$
\begin{aligned}
\overline{x_{A}} & =\int_{0}^{\infty} x P(x) d x \\
& =\frac{1}{\mu}=L,
\end{aligned}
$$

as with any classical collision problem. However, given that the maximum lifetime of a given entity is $\tau$, the maximum possible displacement, $\alpha$, of an entity will be given by

$$
\alpha=\frac{1}{2} g \tau^{2}
$$

The average displacement of such an entity would then be given by

$$
\overline{\mathrm{x}_{\mathrm{A}}}=\int_{0}^{\alpha} \mathrm{x} \mathrm{P}(\mathrm{x}) \mathrm{dx}+\alpha \int_{\alpha}^{\infty} \mathrm{P}(\mathrm{x}) \mathrm{dx}
$$

where the integral in the $2^{\text {nd }}$ term is equal to the probability that the entity does not interact with another entity before a time $\tau$ has passed. Evaluating the integrals of equation (24) results in

$$
\overline{\mathrm{x}_{\mathrm{A}}}=\frac{1}{\mu}\left(1-\mathrm{e}^{-\mu \alpha}\right)
$$

For the case of the entities being displaced in a weak gravitational field, $\mu \alpha<<1$, where the probability of an interaction before a time $\tau$ has passed is very low, equation (25) simplifies to

$$
\overline{\mathrm{X}_{\mathrm{A}}}=\frac{1}{\mu}(1-(1-\mu \alpha+\ldots))=\alpha,
$$


and the entities average displacement is determined by how far the entities can travel in a time equal to their maximum lifetime. For the case of the entities being displaced in a strong gravitational field, $\mu \alpha \gg>1$, where the probability of an interaction before a time $\tau$ has passed is very high, equation (25) simplifies to

$$
\overline{\mathrm{x}_{\mathrm{A}}}=\frac{1}{\mu}=\mathrm{L}
$$

and the entities average displacement is determined by their mean free path length. The BeerLambert law does therefore lead to the expected average displacement of the entities in both a weak and a strong gravitational field.

In equation (16) it is the value of $\overline{x_{A}{ }^{3 / 2}}$ that is wanted. This will be given by

$$
\begin{aligned}
\overline{\mathrm{x}_{\mathrm{A}}^{3 / 2}} & =\int_{0}^{\alpha} \mathrm{x}_{\mathrm{A}}^{3 / 2} \mathrm{P}(\mathrm{x}) \mathrm{dx}+\alpha^{3 / 2} \int_{\alpha}^{\infty} \mathrm{P}(\mathrm{x}) \mathrm{dx} \\
& =\frac{\sqrt{\alpha}}{\mu} \frac{3}{2}\left(\gamma-\mathrm{e}^{-\alpha \mu}\right)
\end{aligned}
$$

where $\gamma$ is given by

$$
\gamma=\frac{\sqrt{\pi}}{2} \frac{\operatorname{erf}(\sqrt{\alpha \mu})}{\sqrt{\alpha \mu}}
$$

Substituting equation (28b) into equation (16) then results in the following expression for the anomalous gravitational field,

$$
\begin{aligned}
\mathrm{g}_{\mathrm{A}} & =\frac{4 \pi \mathrm{G}}{3 \mathrm{c}^{2}} \mathrm{NE}_{1} \sqrt{\frac{2}{\mathrm{~g}}} \frac{\sqrt{\alpha}}{\mu} \frac{3}{2}\left(\gamma-\mathrm{e}^{-\alpha \mu}\right) \\
& =\frac{4 \pi \mathrm{G}}{3 \mathrm{c}^{2}} \mathrm{NE}_{1} \frac{\tau}{\mu} \frac{3}{2}\left(\gamma-\mathrm{e}^{-\alpha \mu}\right) .
\end{aligned}
$$

Equation (30b) can be further simplified by finding an expression for the attenuation coefficient $\mu$. By equations (21) and (14), the attenuation coefficient $\mu$ can be expressed as

$$
\mu=\frac{1}{2} \mathrm{~N} \sigma \sqrt{\frac{2}{\mathrm{~g}}} \overline{\mathrm{x}_{\mathrm{A}}^{1 / 2}}
$$

where

$$
\begin{aligned}
\overline{\mathrm{x}_{\mathrm{A}}{ }^{1 / 2}} & =\int_{0}^{\alpha} \mathrm{x}_{\mathrm{A}}^{1 / 2} \mathrm{P}(\mathrm{x}) \mathrm{dx}+\alpha^{1 / 2} \int_{\alpha}^{\infty} \mathrm{P}(\mathrm{x}) \mathrm{dx} \\
& =\sqrt{\alpha} \gamma .
\end{aligned}
$$


Therefore, by equations (31) and (32b), the attenuation coefficient can be expressed as

$$
\mu=\frac{1}{2} \mathrm{~N} \sigma \tau \gamma \text {. }
$$

By substituting equation (33) into equation (30b) the anomalous gravitational field due to the entities can thereby be expressed as

$$
\mathrm{g}_{\mathrm{A}}=\frac{4 \pi \mathrm{G}}{\mathrm{c}^{2}} \frac{\mathrm{E}_{1}}{\sigma}\left(1-\frac{\mathrm{e}^{-\alpha \mu}}{\gamma}\right)
$$

where $\alpha$ is given by equation (23), $\mu$ is given by equation (33) and $\gamma$ is given by equation (29). The variable $\alpha$ and therefore the variables $\gamma$ and $\mu$ are all functions of $g$. Therefore equation (34) gives the relationship between the anomalous gravitational field $\mathrm{g}_{\mathrm{A}}$ and the total gravitational field $g$.

Equation (34) is a transcendental equation and therefore $\mathrm{g}_{\mathrm{A}}$ cannot be expressed as an explicit function of $\mathrm{g}$. However, the relationship between $\mathrm{g}_{\mathrm{A}}$ and $\mathrm{g}$ as given by equation (34) can be expressed as a series expansion. This can be shown as follows. Starting from the series expansions for the error and exponential functions the following expansions can be derived;

$$
\gamma=1-\frac{1}{3}(\alpha \mu)+\frac{1}{10}(\alpha \mu)^{2}-\mathcal{O}\left[(\alpha \mu)^{3}\right]
$$

and

$$
\left(1-\frac{\mathrm{e}^{-\alpha \mu}}{\gamma}\right)=\frac{2}{3}\left[(\alpha \mu)-\frac{4}{15}(\alpha \mu)^{2}+\mathcal{O}\left[(\alpha \mu)^{3}\right]\right]
$$

In addition, by equations (33) and (35);

$$
\begin{aligned}
\alpha \mu & =\frac{1}{2} \mathrm{~N} \sigma \tau \alpha \gamma \\
& =\left(\alpha \mu_{0}\right)-\frac{1}{3}\left(\alpha \mu_{0}\right)^{2}+\mathcal{O}\left[\left(\alpha \mu_{0}\right)^{3}\right]
\end{aligned}
$$

where

$$
\mu_{0}=\frac{1}{2} \mathrm{~N} \sigma \tau
$$

is the attenuation coefficient in the weak field limit, i.e. setting $\overline{t_{E}}$ in equation (21) equal to $\tau$. By substituting equation (37b) into equation (36) and then equation (36) into equation (34), the following series expansion for $\mathrm{g}_{\mathrm{A}}$ is thereby obtained;

$$
\mathrm{g}_{\mathrm{A}}=\frac{8 \pi \mathrm{G}}{3 \mathrm{c}^{2}} \frac{\mathrm{E}_{1}}{\sigma}\left(\left(\alpha \mu_{0}\right)-\frac{3}{5}\left(\alpha \mu_{0}\right)^{2}+\mathcal{O}\left[\left(\alpha \mu_{0}\right)^{3}\right]\right)
$$




$$
=\frac{8 \pi G}{3 c^{2}} \frac{E_{1}}{\sigma}\left(\left(\frac{g}{g_{0}}\right)-\frac{3}{5}\left(\frac{g}{g_{0}}\right)^{2}+\mathcal{O}\left[\left(\frac{g}{g_{0}}\right)^{3}\right]\right)
$$

where the constant $\mathrm{g}_{0}$, with units of $\mathrm{m} \mathrm{s}^{-2}$, is defined as

$$
\mathrm{g}_{0}=\frac{2}{\mu_{0} \tau^{2}} .
$$

In the far field of galaxies, where $\mathrm{g} \ll \mathrm{g}_{0}$, the observed galactic rotational curves would indicate that the induced gravitational field is the dominant contributor to the total gravitational field. Therefore for $g<<g_{0}$, the anomalous gravitational field $g_{A} \rightarrow g$. In this limit it then follows from equation $(39 b)$ that

$$
\mathrm{g}_{0}=\frac{8 \pi \mathrm{G}}{3 \mathrm{c}^{2}} \frac{\mathrm{E}_{1}}{\sigma}
$$

By substituting equation (41) into equation (39b) the following second order weak field approximation for the dependence that $g_{A}$ has on $g$ is then found;

$$
\frac{\mathrm{g}_{\mathrm{A}}}{\mathrm{g}_{0}} \cong \frac{\mathrm{g}}{\mathrm{g}_{0}}-\frac{3}{5}\left(\frac{\mathrm{g}}{\mathrm{g}_{0}}\right)^{2}
$$

Equation (42) is of the form of equation (4) and as is shown in Penner [21] a functional dependence of this form leads to the baryonic Tully-Fisher relationship.

To derive the baryonic Tully-Fisher relationship from equation (42) the substitution of $g$ $=\mathrm{g}_{\mathrm{M}}+\mathrm{g}_{\mathrm{A}}$ into the first order term of equation (42) is made, resulting in

$$
\frac{\mathrm{g}_{\mathrm{A}}}{\mathrm{g}_{0}} \cong \frac{\mathrm{g}_{\mathrm{A}}}{\mathrm{g}_{0}}+\frac{\mathrm{g}_{\mathrm{M}}}{\mathrm{g}_{0}}-\frac{3}{5}\left(\frac{\mathrm{g}}{\mathrm{g}_{0}}\right)^{2}
$$

which then simplifies to

$$
\mathrm{g}_{\mathrm{M}} \cong \frac{3}{5} \frac{\mathrm{g}^{2}}{\mathrm{~g}_{0}}
$$

Then by substituting for $\mathrm{g}_{\mathrm{M}}$ from equation (1) and equating the total gravitational field $\mathrm{g}$ to the centripetal acceleration of an orbiting mass, i.e. $\mathrm{v}^{2} / \mathrm{r}$, the following relationship is found between the baryonic mass of a given galaxy and the weak or far field value for the orbiting speed of the stars;

$$
\mathrm{M}=\frac{3}{5 \mathrm{Gg}_{0}} \mathrm{v}^{4}
$$

Equation (45) is in agreement with the baryonic Tully-Fisher relationship. The hypotheses and the model presented in this paper leads therefore to the baryonic Tully-Fisher relationship. The details of the model and the approximations used will not affect this result. As discussed in 
Penner [21], if a gravitational field induces a contribution from the cosmos then in general $\mathrm{g}_{\mathrm{A}}$ is a function of $\mathrm{g}$ and can be expanded in a form as per equation (39b). This in turn leads to equation (44) which results in the dependence between $\mathrm{M}$ and $\mathrm{v}$ as given by the baryonic Tully-Fisher relationship.

The value for the constant $\mathrm{g}_{0}$ in equation (45) can be determined by equating the coefficient of equation (45) to the coefficient of the observed baryonic Tully-Fisher relationship, equation (3). The resulting value for $\mathrm{g}_{0}$ is

$$
\mathrm{g}_{0}=1.29 \times 10^{-10} \mathrm{~m} \mathrm{~s}^{-2} \text {. }
$$

In a strong gravitational field where $g>g_{0}$ and $\alpha \mu \gg>1$, equations (34) and (41) indicate then that $g_{\mathrm{A}}$ approaches a constant value of

$$
\begin{aligned}
g_{A} & =\frac{4 \pi G}{c^{2}} \frac{E_{1}}{\sigma} \\
& =\frac{3}{2} g_{0} .
\end{aligned}
$$

Therefore in the strong field realm of the Pioneer anomaly, by equations (46) and (47b), $\mathrm{g}_{\mathrm{A}}$ is approximately constant with a value of

$$
\mathrm{g}_{\mathrm{A}}=1.94 \times 10^{-10} \mathrm{~m} \mathrm{~s}^{-2} \text {. }
$$

Although this value is smaller than the observed value for the Pioneer anomaly, as given by equation (2), it is still encouragingly close. This is especially so given that the range of $\mathrm{g}$ being considered in going from the realm of the baryonic Tully-Fisher relationship to the realm of the Pioneer anomaly is over five orders of magnitude.

To explain the difference between the models' value, equation (48), and the observed value for the Pioneer anomaly, equation (2), the assumption that entities exist only with energies $\pm \mathrm{E}_{1}$ needs to be reconsidered. Consider the possibility where entities of both energies $\pm \mathrm{E}_{1}$ and $\pm \mathrm{E}_{2}$ exist. In order to keep things relatively simple, it will be taken that $\mathrm{N}$, the rate at which entities come into existence per unit volume, and $\sigma$, the cross-section for the interactions, is the same for both the $\pm \mathrm{E}_{1}$ entities and the $\pm \mathrm{E}_{2}$ entities. Also only the interactions between entities of equal and opposite energy are of concern, i.e. interactions which result in the removal of the entities. As such the contribution of the $\pm \mathrm{E}_{1}$ entities and the $\pm \mathrm{E}_{2}$ entities are independent of each other and the net anomalous gravitational field will be given by

$$
\mathrm{g}_{\mathrm{A}}=\mathrm{g}_{\mathrm{A} 1}+\mathrm{g}_{\mathrm{A} 2}
$$

where by equation (34),

$$
g_{A 1}=\frac{4 \pi G}{c^{2}} \frac{E_{1}}{\sigma}\left(1-\frac{e^{-\alpha_{1} \mu_{1}}}{\gamma_{1}}\right)
$$


and

$$
\mathrm{g}_{\mathrm{A} 2}=\frac{4 \pi G}{\mathrm{c}^{2}} \frac{\mathrm{E}_{2}}{\sigma}\left(1-\frac{\mathrm{e}^{-\alpha_{2} \mu_{2}}}{\gamma_{2}}\right)
$$

In the weak field approximation, equations (50a) and (50b) can be shown to simplify to

$$
\mathrm{g}_{\mathrm{A} 1} \cong \frac{\mathrm{E}_{2}^{2}}{\mathrm{E}_{1}{ }^{2}+\mathrm{E}_{2}{ }^{2}} \mathrm{~g}_{\mathrm{A}}
$$

and

$$
\mathrm{g}_{\mathrm{A} 2} \cong \frac{\mathrm{E}_{1}{ }^{2}}{\mathrm{E}_{1}{ }^{2}+\mathrm{E}_{2}{ }^{2}} \mathrm{~g}_{\mathrm{A}}
$$

Consider now as an example the case where $\mathrm{E}_{2}=3.5 \mathrm{E}_{1}$. For this example, equations (51a) and (51b) result in

$$
\mathrm{g}_{\mathrm{A} 1} \cong \frac{12.25}{13.25} \mathrm{~g}_{\mathrm{A}}
$$

and

$$
\mathrm{g}_{\mathrm{A} 2} \cong \frac{1}{13.25} \mathrm{~g}_{\mathrm{A}}
$$

Therefore, in the weak field limit where the baryonic Tully-Fisher relationship holds, the contribution to $\mathrm{g}_{\mathrm{A}}$ provided by the $\pm \mathrm{E}_{1}$ entities will dominate. The second order coefficient in equation (42) will remain at approximately $3 / 5$ and the value for $g_{0}$ will stay at approximately the value as given by equation (46).

In a strong field, $\alpha \mu>>1$, equations (50a) and (50b) simplify to

$$
\mathrm{g}_{\mathrm{A} 1}=\frac{4 \pi \mathrm{G}}{\mathrm{c}^{2}} \frac{\mathrm{E}_{1}}{\sigma}=\frac{3}{2} \mathrm{~g}_{0}
$$

and

$$
\mathrm{g}_{\mathrm{A} 2}=\frac{4 \pi \mathrm{G}}{\mathrm{c}^{2}} \frac{\mathrm{E}_{2}}{\sigma}=\frac{\mathrm{E}_{2}}{\mathrm{E}_{1}}\left(\frac{3}{2} \mathrm{~g}_{0}\right)
$$

Therefore, in the strong field limit, where the Pioneer anomaly holds, the contribution to $\mathrm{g}_{\mathrm{A}}$ by the $\pm \mathrm{E}_{2}$ entities will dominate. For the example with $\mathrm{E}_{2}=3.5 \mathrm{E}_{1}$ the contribution due to the higher energy entities will be 3.5 times greater than the contribution due to the lower energy entities. and the value of $g_{A}$ will approach a value of

$$
\mathrm{g}_{\mathrm{A}}=\frac{3}{2} \mathrm{~g}_{0}+3.5\left(\frac{3}{2} \mathrm{~g}_{0}\right)=6.75 \mathrm{~g}_{0}
$$




$$
=8.71 \times 10^{-10} \mathrm{~m} \mathrm{~s}^{-2}
$$

The example provided, with $\mathrm{E}_{2}=3.5 \mathrm{E}_{1}$ and $\mathrm{N}$ being the same for both the $\pm \mathrm{E}_{1}$ and the $\pm \mathrm{E}_{2}$ entities, does therefore lead to agreement with both the baryonic Tully-Fisher relationship and the observed value of the Pioneer anomaly. However, this is to be taken as no more than an example. Other distributions of the possible entities energy values could also be found to agree with the observations. In general, as demonstrated by the example, if more than one entity energy exists, the strong field value will exceed the value given by equation (48).

From the model presented some of the details of the entities can be determined. Expressing the interaction cross-section $\sigma$ as equal to $\pi r_{0}^{2}$, where $r_{0}$ is the range of the interaction, the following inequality would be expected to hold;

$$
\mathrm{N} \tau<\left(\frac{4}{3} \pi \mathrm{r}_{0}^{3}\right)^{-1}
$$

where $\mathrm{N} \tau$ is the maximum entity density, i.e. the density of entities in the weak field limit. Equation (55) basically states that the average separation of the entities is greater than their interaction range. Equations (55), (38), (40), (41), and (6) along with the value of $\mathrm{g}_{0}$ as given by equation (46) then lead to the following inequalities;

$$
\begin{aligned}
& \mathrm{r}_{0}<2.8 \times 10^{-23} \mathrm{~m}, \\
& \sigma<2.4 \times 10^{-45} \mathrm{~m}^{2}, \\
& \mathrm{E}_{1}<4.9 \times 10^{-29} \mathrm{~J}, \text { and } \\
& \tau_{1}>1.1 \times 10^{-6} \mathrm{~s} .
\end{aligned}
$$

In Penner [21] it was conjectured that the entities were bound particle-antiparticle pairs. From equation $(56 \mathrm{~b})$ it is seen that the speculation given by the author in [21] on the nature of the entities must be incorrect. Given the cross section for the interaction, equation (56b), the entities would appear to be single uncharged particles which must only interact via the weak force. At this time no further speculation will be offered regarding the nature of these entities.

\section{Conclusion}

The theory of induced gravitational contribution from the cosmos that is presented in this paper leads to both the baryonic Tully-Fisher relationship and the Pioneer anomaly. This theory ties the two gravitational anomalies to each other, with the coefficient of the baryonic TullyFisher relationship determining the constant value that $\mathrm{g}_{\mathrm{A}}$ approaches in a strong gravitational field. The approximations and simplifications presented are substantial. However, given that the 
baryonic Tully-Fisher relationship and the Pioneer anomaly do fall out naturally from the proposed theory it would seem that this theory is pointing in the right direction.

An issue that needs to be addressed is why the value of the observed Pioneer anomaly drops towards zero as one approaches the Sun. The rapid rate at which it drops may be a clue. One physical property that rapidly changes with distance from the Sun is the energy density of the solar magnetic field. The energy density of the magnetic field of a magnetic dipole, such as the Sun's, drops off with distance as $\mathrm{r}^{-6}$. The solar magnetic field density therefore increases by a factor of 64 as the distance from the Sun is reduced from 20 AU's to 10 AU's. If an electromagnetic field inhibits the coming into existence of the proposed entities of the model, reduces the lifetime of the entities or reduces the amount of their displacement, then a rapid drop in the value of $g_{A}$ as the Sun is approached would be expected. Interestingly, if the solar magnetic field is responsible for a reduction in the value for $g_{A}$ then it would be expected that the value of $g_{A}$ within our solar system would not only vary with the distance from the Sun but also on the orientation with respect to the solar magnetic field. However, more work is required to explain why the Pioneer anomaly drops rapidly to zero within the inner solar system.

In the model presented in this paper, the positive net energy entities shift towards a gravitational source while the negative entities shift away, with the result that the energy density of the vacuum surrounding a given mass is positive. A positive energy density corresponds to having more positive energy entities than negative energy entities within a given volume. If only a single gravitational source is present and the cosmos is bounded then, as with the analogous case of a dielectric with an embedded charge, the bounding surface would have a net negative energy and overall the interior vacuum and the bounding surface would have a net energy of zero. Of course, the Universe does not have a bounding surface and multiple gravitational sources are present. There then must be regions where the vacuum energy density is positive, i.e. in the regions surrounding masses, and regions where the vacuum energy density is negative. It is speculated that the regions where the vacuum energy density is negative corresponds to the voids between galactic clusters. If this is the case, these voids with their net negative energy would be expected to repel mass. Therefore, while on the one hand the energy polarization of the vacuum leads to stronger gravitational fields surrounding matter and thereby leads to greater and faster accumulations of mass, on the other hand the energy polarization of the vacuum also leads to these accumulations of mass being repelled away from the voids and thereby away from each other. How this may be related to the observed acceleration of galactic clusters away from each other, which is currently explained by the concept of dark energy, will not be speculated on at this time. 


\section{References:}

[1] J.D. Anderson, P.A. Laing, A.S. Lau, M.M. Nieto, and S.G. Turyshev, Phys. Rev. Lett. 81, 2858, (1998)

[2] J.D. Anderson, P.A. Laing, A.S. Lau, M.M. Nieto, and S.G. Turyshev, Phys. Rev. D 65 082004/1-50, (2002)

[3] S.G. Turyshev and V.T. Toth, arxiv.org/abs/1001.3686, (2010)

[4] W.M. Folkner, "Relativistic aspects of the JPL planetary ephemeris", in S.A. Klioner, P.K. Seidelmann, and M.H. Soffel, eds., Relativity in Fundamental Astronomy:

Dynamics, Reference Frames, and Data Analysis, Proceedings IAU Symposium No. 261, 22 April- 1 May 2009, Virginia Beach, VA, USA, Proc. IAU, vol. 261, pp. 155-158

(Cambridge University Press, Cambridge, 2010). [DOI][ADS]

[5] R.B. Tully and J.R. Fisher, A\&A, 54, 661, (1977)

[6] S.S. McGaugh, J.M. Schombert, G.D. Bothun, and W.J. DeBlok, ApJ, 533, L99, (2000)

[7] F. Zwicky, Helv. Phys. Acta 6, 110, (1933)

[8] M. Milgrom, New Astron. Rev. 46, 741, (2002)

[9] M. Milgrom, Astrophys. J. 270, 365, (1983)

[10] M.Milgrom, Ann. Phys. 229, 384, (1994)

[11] M. Milgrom, Phys. Lett. A, 253, 273, (1999)

[12] M.E. McCulloch, Mon. Not. Roy. Astr. Soc. 376, 338, (2007)

[13] J.W. Moffat, J. Cosmol. Astropart. Phys. JCAP05(2005)003

[14] J.W. Moffat, J. Cosmol. Astropart. Phys. JCAP03(2006)004

[15] J.R. Brownstein and J.W. Moffat, Astrophys. J. 636, 721, (2006)

[16] J.W. Moffat and V.T. Toth, Class. Quant. Grav. 26, 5002, (2009)

[17] J.R. Brownstein and J.W. Moffat, Class. Quant. Grav. 23, 3427, (2006)

[18] L. Blanchet, Class. Quant. Grav. 24, 3529, astro-ph/0605637, (2007)

[19] L. Blanchet, Class. Quant. Grav. 24, 3541, gr-qc/0609121, (2007)

[20] L. Blanchet and A. LeTiec, arxiv.org/abs/0804.3518, (2008) 
Induced energy polarization of the vacuum as the source of the baryonic Tully-Fisher relationship and the Pioneer anomaly

[21] A. R. Penner, Can. J. Phys. 89, 841, (2011)

[22] Lorrain, P., and Corson, D., Ch. 3, Electromagnetic Fields and Waves 1970, (2 $2^{\text {nd }}$ ed; San Fransisco) 